\title{
Excited State Transitions in Double Beta Decay: A brief Review
}

\author{
Björn Lehnert ${ }^{1, a}$ \\ ${ }^{1}$ Institut für Kern- und Teilchenphysik, Technische Universität Dresden, Dresden, Germany
}

\begin{abstract}
Neutrinoless double beta decays requires lepton number violation and provide an important probe for physics beyond the Standard Model. Nuclear matrix elements (NME) are needed to connect the half-life of the decay with the neutrino mass. The calculation of these NME depends on nuclear models and is subject to large uncertainties. $0 v \beta \beta$ and $2 v \beta \beta$ decays can also occur into excited states. Especially the $2 v \beta \beta$ excited state transitions are accessible in current experiments and together with the ground state transitions provide an important cross check for nuclear model calculations.

This contribution will present the current experimental status of excited state transitions in double beta decay isotopes.
\end{abstract}

\section{Introduction}

Double beta decays are second order weak nuclear decays. Their observation is only feasible in nuclear configurations where two consecutive single beta decays are energetically forbidden or otherwise strongly suppressed. The two-neutrino double beta $(2 v \beta \beta)$ decay is a Standard Model process which has been observed in 13 isotopes with half-lives around $10^{18-21} \mathrm{yr}$. The neutrino-less double beta $(0 v \beta \beta)$ decay is lepton number violating (LNV) and requires massive neutrino mass eigenstates and the Majorana nature of neutrinos:

$$
\begin{array}{ll}
2 v \beta^{-} \beta^{-}: & (Z, A) \rightarrow(Z+2, A)+2 e^{-}+2 \bar{v}_{e} \\
0 v \beta^{-} \beta^{-}: & (Z, A) \rightarrow(Z+2, A)+2 e^{-}
\end{array}
$$

The experimental signature is the energy of two $e^{-}$which show a continues spectrum up to the $\mathrm{Q}$-value for the $2 v \beta \beta$ mode and a discrete electron sum energy in case of the $0 v \beta \beta$ mode. The $0 v \beta \beta$ decay has not been observed and the calculation of the half-life requires two model dependent assumptions: (1) the LNV process and (2) a nuclear model describing the nuclear matrix element (NME). The standard interpretation of (1) is the light Majorana neutrino exchange. In this interpretation, $T_{1 / 2}^{0 v}$ is proportional to the square of the inverse Majorana neutrino mass $\left|m_{e e}\right|^{-2}$. Instead, the calculation of the $2 v \beta \beta$ half-life requires only the knowledge of the NME:

$$
\begin{aligned}
& 2 v \beta^{-} \beta^{-}:\left(T_{1 / 2}^{2 v}\right)^{-1}=G^{2 v} \cdot\left|\mathcal{M}^{2 v}\right|^{2} \\
& 0 v \beta^{-} \beta^{-}:\left(T_{1 / 2}^{0 v}\right)^{-1}=G^{0 v} \cdot\left|\mathcal{M}^{0 v}\right|^{2} \cdot\left|m_{e e}\right|^{2}
\end{aligned}
$$

Here, $G^{2 / 0 v}$ is the phase space factor (PSF) and $\mathcal{M}^{2 / 0 v}$ the NME of the decay mode. The calculation of the PSF is relatively straight forward. As a leading term approximation,

\footnotetext{
a e-mail: bjoern.lehnert@tu-dresden.de
}

$G^{2 v}$ is proportional to $E^{11}$ whereas $G^{0 v}$ is proportional to $E^{5}$. Current calculations considering nuclear and atomic corrections are performed e.g. in [1]. The NMEs $\mathcal{M}^{2 v}$ and $\mathcal{M}^{0 v}$ are inherently different. $2 v \beta \beta$ decays are only possible via intermediate $1^{+}$states whereas $0 v \beta \beta$ also go via other intermediate states. The $2 v \beta \beta$ transitions can be used to validate calculations in a specific nuclear model framework. In addition, free or unknown parameters that enter the NME calculations can be constrained as e.g. the quenching of $g_{A}$ in nuclear matter.

DBD decays can also occur into excited states of the daughter nucleus. This extends the system of Eq. $(3,4)$. Excited state transitions have a significantly reduced rate due to a smaller phase space. Furthermore, final states other than $0^{+}$are suppressed by spin combinations. The relaxation of the daughter nucleus is accompanied by a gamma cascade which provides an enhanced experimental signature. The excited state transition with the largest rate is expected to be the first excited $0_{1}^{+}$state for $2 v \beta \beta$. In the specific nuclear configuration of DBD isotopes, the ground states of the mother and daughter isotope are $0_{\mathrm{g} . \mathrm{s}}^{+}$ states. Typically, the first excited state in the daughter is a $2^{+}$state. Excited $0^{+}$states cannot directly decay into the $0_{\text {g.s. }}^{+}$and cascade via the $2^{+}$state while emitting two gammas. This is illustrated for the case of ${ }^{76} \mathrm{Ge}$ in Fig. 1.

The properties of the most prominent DBD isotopes are listed in Tab. 1 showing the Q-value, the excitation level of $0_{1}^{+}$as well as the most prominent $\gamma$-lines for experimental searches.

\section{Experimental Considerations}

A number of past review articles contain experimental and theoretical data on excited state transitions. A complete list up to 2002 is published in [15]. A collection of excited 
Table 1. Excited state structure in important DBD isotopes. The columns denote: nuclide and transition, Q-value of the decay, energy level of first excited $0^{+}$state and the observable $\gamma$-lines in the experimental signature. $\gamma$-lines are ordered as $0_{1}^{+}-2_{1}^{+}$, $2_{1}^{+}-0_{\mathrm{g} . \mathrm{s} .}^{+}$transition. For ${ }^{130} \mathrm{Te}$ there are multiple decay branches going via two $2^{+}$states. The branches are ordered with decreasing probability. All information from [2] if not otherwise noted.

\begin{tabular}{|c|c|c|c|}
\hline Transition & $\begin{array}{l}\text { Q-value } \\
{[\mathrm{keV}]}\end{array}$ & $\begin{array}{r}0_{1}^{+} \text {state } \\
{[\mathrm{keV}]}\end{array}$ & $\begin{array}{r}\gamma \text {-lines } \\
{[\mathrm{keV}]}\end{array}$ \\
\hline${ }^{48} \mathrm{Ca} \rightarrow{ }^{48} \mathrm{Ti}$ & $4262.96 \pm 0.84[3]$ & 2997.22 & 2013.66, 983.53 \\
\hline${ }_{32}^{76} \mathrm{Ge} \rightarrow{ }_{34}^{20} \mathrm{Se}$ & $2039.006 \pm 0.050[4]$ & 1122.28 & $563.18,559.10$ \\
\hline${ }_{34}^{82} \mathrm{Se} \rightarrow{ }_{36}^{82} \mathrm{Kr}$ & $2997.9 \pm 0.3[5]$ & 1487.60 & $711.2,776.52$ \\
\hline${ }_{40}^{96} \mathrm{Zr} \rightarrow{ }_{42}^{96} \mathrm{Mo}$ & $3347.7 \pm 2.2[6]$ & 1148.13 & $369.80,778.22$ \\
\hline${ }_{42}^{400} \mathrm{Mo} \stackrel{42}{\rightarrow}{ }_{44}^{100} \mathrm{Ru}$ & $3034.40 \pm 0.17[7]$ & 1130.32 & $590.79,539.51$ \\
\hline${ }_{46}^{42} \mathrm{Pd} \rightarrow{ }_{48}^{110} \mathrm{Cd}$ & $2017.85 \pm 0.64[8]$ & 1473.07 & $815.31,657.76$ \\
\hline${ }_{48}^{116} \mathrm{Cd} \rightarrow{ }_{50}^{416} \mathrm{Sn}$ & $2813.50 \pm 0.13[9]$ & 1756.86 & $463.25,1293.56$ \\
\hline${ }_{50}^{424} \mathrm{Sn} \rightarrow{ }_{52}^{124} \mathrm{Te}$ & $2292.64 \pm 0.39[10]$ & 1657.28 & $1054.55,602.73$ \\
\hline${ }_{52}^{130} \mathrm{Te} \rightarrow{ }_{54}^{130} \mathrm{Xe}$ & $2527.518 \pm 0.013[11]$ & 1793.52 & $\begin{array}{r}1257.5,536.07 \\
671.9,586.05,536.07\end{array}$ \\
\hline & & & $671.9,1122.15$ \\
\hline${ }_{54}^{136} \mathrm{Xe} \rightarrow{ }_{56}^{136} \mathrm{Ba}$ & $2457.83 \pm 0.37[12][13]$ & 1578.99 & $760.49,818.51$ \\
\hline${ }_{60}^{150} \mathrm{Nd} \rightarrow{ }_{62}^{150} \mathrm{Sm}$ & $3371.38 \pm 0.20[14]$ & 740.46 & $406.51,333.96$ \\
\hline
\end{tabular}

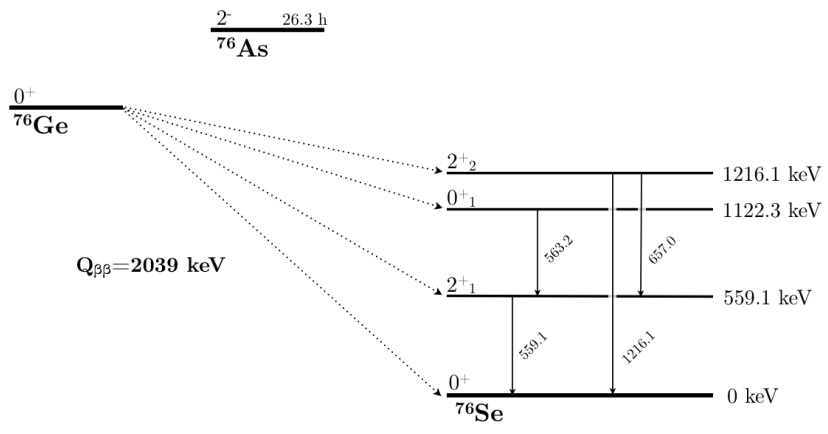

Figure 1. Decay scheme of ${ }^{76} \mathrm{Ge}$ serving as an example for a typical configuration of double beta decays into excited states.

state results up to 2007 is published in [16]. A combination of half-lifes from multiple measurements of the same isotope up to 2010 is presented in [17]. A few new experimental results were achieved since then and will be briefly described here.

The experimental search for DBD excited state transitions can be distinguished into two approaches: (1) with gamma spectroscopy of a DBD target using HPGe detectors or (2) as secondary analysis in large scale $0 v \beta \beta$ decay experiments.

The first approach is a dedicated search for de-excitation $\gamma$-lines and cannot distinguish between the $2 v \beta \beta$ and the $0 v \beta \beta$ mode. The only two observations of excited state transitions in ${ }^{100} \mathrm{Mo}$ and ${ }^{150} \mathrm{Nd}$ have been achieved with gamma spectroscopy. The half-lives have been measured with $T_{1 / 2}=(7.5 \pm 1.2) \cdot 10^{20} \mathrm{yr}$ [18] and $T_{1 / 2}=$ $\left(1.33_{-0.36}^{+0.63}\right) \cdot 10^{20}$ yr [19] in ${ }^{100} \mathrm{Mo}$ and ${ }^{150} \mathrm{Nd}$ respectively. Recently, first limits in ${ }^{110} \mathrm{Pd}$ were obtained with gamma spectroscopy. A $0.8 \mathrm{~kg}$ palladium sample was investigated in the Felsenkeller low-level underground laboratory in Dresden, Germany [20] and in the HADES underground facility of IRMM Mol, Belgium [21]. The best half-life limit was found to be $>2.0 \cdot 10^{20} \mathrm{yr}(95 \%$ C.L.).
The second approach profits from the low background infrastructure and large target materials in $0 v \beta \beta$ experiments. Many of these experiments are segmented in individual detectors and use anti-coincidence vetoes between these segments to distinguish background from $0 v \beta \beta$ events; the electrons of a $0 v \beta \beta$ decay deposit their energy in a small volume whereas $\gamma$-background often results in distributed energy depositions due to scattering. The anti-coincidence vetoes for $0 v \beta \beta$ can be used searching for coincides between $\beta \beta$ and $\gamma$ energies of excited state transitions. In homogeneous DBD experiments as e.g. LXe in EXO-200 or NEXT, pulse shapes can be used to distinguish between single-site and multi-site energy depositions.

New experimental results are available using current $0 v \beta \beta$ experiments: CUORICINO uses $11.3 \mathrm{~kg} \mathrm{TeO}$ in 62 bolometer crystals at LNGS and found a new best limit for $2 v \beta \beta 0_{1}^{+}$in ${ }^{130} \mathrm{Te}$ as $T_{1 / 2}>1.3 \cdot 10^{23} \mathrm{yr}(90 \% \mathrm{CL})$ [22]. CUORICINO is a prototype tower of the CUORE detector which will have 19 similar towers with a target mass of $204 \mathrm{~kg}{ }^{130} \mathrm{Te}$ and a total segmentation of 988 . CUORE will be able to strongly improve the sensitivity for this decay mode in the near future.

Another recent results comes from the EXO-200 experiment investigating ${ }^{136} \mathrm{Xe}$ with a LXe TPC in the WIPP near Carlsbad, New Mexico. A large fiducial target mass of $80 \mathrm{~kg}$ enriched LXe is achieved; however, nonsegmentation and poor energy resolution increase the difficulty to identify the excited state event topologies. A pulse shape quantifier is used to investigate multi-site events. A fit is performed over a wide energy range resulting in a new best limit for $2 v \beta \beta 0_{1}^{+}$in ${ }^{136} \mathrm{Xe}$ of $T_{1 / 2}>1.2 \cdot 10^{23} \mathrm{yr}$ $(90 \% \mathrm{CL})$ is obtained [23].

\section{Theoretical Considerations}

The half-life for excited state transitions can be calculated directly via Eq. 3. Various direct calculations have been performed in the past which are, however, largely older than 10 years. An alternative is to use the ratio of NMEs and PSFs for the $0_{1}^{+}$and $0_{\text {g.s. }}^{+}$transition and scale that ratio with the $0_{\mathrm{g} . \mathrm{s} .}^{+}$half-life:

$$
\mathcal{R}=\frac{T_{1 / 2}^{0_{1}^{+}}}{T_{1 / 2}^{\text {g.s. }}}=\frac{\Gamma_{\text {g.s. }}}{\Gamma_{0_{1}^{+}}}=\frac{\left(\mathcal{M}_{\text {g.s. }}\right)^{2} \cdot G_{\text {g.s. }}}{\left(\mathcal{M}_{0_{1}^{+}}\right)^{2} \cdot G_{0_{1}^{+}}} .
$$

The ratio of NMEs has the advantage than some theoretical uncertainty cancel out and the excited state half-life prediction becomes more reliable. This approach can be used with a systematical and intra-comparable calculation of NMEs as is e.g. performed with IBM-2 model [24].

Tab. 2 shows the experimental half-lives and half-life limits for the $2 v \beta \beta 0_{\mathrm{g} . \mathrm{s} .}^{+}$and $0_{1}^{+}$transitions for prominent DBD isotopes. The third and fourth column show a list of direct calculations for $0_{1}^{+}$using QRPA. The prediction in the last column is calculated using the ration in Eq. 5 with NMEs from [24], PSFs from [1] and $T_{1 / 2}^{\text {g.s. }}$ from column 2.

The theoretical predictions from QRPA and IBM-2 often disagree strongly. The tendency is that IBM-2 predicts longer half-lives. Many of the older QRPA predictions 
Table 2. Current data for excited state transitions. Shown are observed half-lives or half-life limits of the ground state and $0_{1}^{+}$ transitions. The last three columns show theoretical predictions: two direct calculations from QRPA and a calculated half-life ratio between $0_{1}^{+}$and $0_{\mathrm{g} . s .}^{+}$scaled with the measured $0_{\mathrm{g} . \mathrm{s} .}^{+}$half-life as described in the text.

\begin{tabular}{|c|c|c|c|c|c|}
\hline $\begin{array}{l}\text { Transition } \\
2 v \beta \beta\end{array}$ & $\begin{array}{l}0_{\mathrm{g} . S .}^{+} \exp \\
T_{1 / 2}[\mathrm{yr}] \\
\end{array}$ & $\begin{array}{c}0_{1}^{+} \exp \\
T_{1 / 2}[\mathrm{yr}]\end{array}$ & $\begin{array}{c}0_{1}^{+} \text {QRPA [26] [27] } \\
T_{1 / 2}[\mathrm{yr}]\end{array}$ & $\begin{array}{c}0_{1}^{+} \text {QRPA [28] } \\
T_{1 / 2} \text { [yr] }\end{array}$ & $\begin{array}{c}0_{1}^{+} \text {IBM-2 }[1,24] \\
T_{1 / 2}[\mathrm{yr}]\end{array}$ \\
\hline${ }_{20}^{48} \mathrm{Ca} \rightarrow{ }_{20}^{48} \mathrm{Ti}$ & $4.4_{-05}^{+0.6} \cdot 10^{19}[17]^{a}$ & $>1.5 \cdot 10^{20}[29]$ & - & - & $1.8 \cdot 10^{23}$ \\
\hline${ }_{32}^{76} \mathrm{Ge} \rightarrow{ }_{34}^{76} \mathrm{Se}$ & $1.84_{-0.10}^{+0.14} \cdot 10^{21}[30]$ & $>6.2 \cdot 10^{21}[31]$ & $(7.5-310) \cdot 10^{21}$ & $4.5 \cdot 10^{21}$ & $6.0 \cdot 10^{24}$ \\
\hline 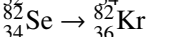 & $9.2 \pm 0.7 \cdot 10^{19}[17]^{a}$ & $>3.0 \cdot 10^{21}[32]$ & $(1.5-3.3) \cdot 10^{21}$ & - & - \\
\hline${ }_{40}^{94} \mathrm{Zr} \rightarrow{ }_{42}^{96} \mathrm{Mo}$ & $2.3 \pm 0.2 \cdot 10^{19}[17]^{a}$ & $>6.8 \cdot 10^{19}[33]$ & $(2.4-2.7) \cdot 10^{21}$ & $3.8 \cdot 10^{21}$ & $2.8 \cdot 10^{24}$ \\
\hline${ }_{42}^{40} \mathrm{Mo} \rightarrow{ }^{400} \mathrm{Ru}$ & $7.1 \pm 0.4 \cdot 10^{18}[17]^{a}$ & $=5.9^{+0.8} \cdot 10^{20}[17]^{a}$ & $1.6 \cdot 10^{21}[34]$ & $2.1 \cdot 10^{21}$ & $2.2 \cdot 10^{22}$ \\
\hline${ }_{46}^{110} \mathrm{Pd} \rightarrow{ }_{48}^{11}$ & $>1 \cdot 10^{17}[35]$ & $>2.0 \cdot 10^{20}[21]$ & $(4.2-9.1) \cdot 10^{23}[36]$ & - & $>1.1 \cdot 10^{22}$ \\
\hline${ }^{6} \mathrm{Cd} \rightarrow{ }_{50}^{116} \mathrm{Sn}$ & $2.8 \pm 0.2 \cdot 10^{19}[17]^{a}$ & $>2.0 \cdot 10^{21}[37]$ & $1.1 \cdot 10^{22}$ & $1.1 \cdot 10^{21}$ & $6.4 \cdot 10^{23}$ \\
\hline${ }_{50}^{124} \mathrm{Sn} \rightarrow{ }_{52}^{124} \mathrm{Te}$ & - & - & $2.7 \cdot 10^{21}$ & - & - \\
\hline${ }_{52}^{130} \mathrm{Te} \rightarrow{ }_{54}^{130} \mathrm{Xe}$ & $6.8_{-1.1}^{+1.2} \cdot 10^{20}[17]^{a}$ & $>1.3 \cdot 10^{23}[22]$ & $(5.1-14) \cdot 10^{22 b}$ & - & $2.2 \cdot 10^{25}$ \\
\hline${ }_{54}^{136} \mathrm{Xe} \rightarrow{ }_{56}^{136} \mathrm{Ba}$ & $2.29 \pm 0.08 \cdot 10^{21 b}$ & $>1.2 \cdot 10^{23}[23]$ & $2.5 \cdot 10^{21}$ & $6.3 \cdot 10^{21}$ & $2.6 \cdot 10^{25}$ \\
\hline${ }_{60}^{150} \mathrm{Nd} \rightarrow{ }_{62}^{150} \mathrm{Sm}$ & $8.2 \pm 0.9 \cdot 10^{18}[17]^{a}$ & $=1.33_{-0.26}^{+0.45} \cdot 10^{20}[17]^{a}$ & - & - & $1.9 \cdot 10^{21}$ \\
\hline
\end{tabular}

${ }^{a}$ world average values

${ }^{b}$ corrected in [38]

are ruled out by recent experimental limit. Most IBM-2 predictions are still below the current experimental sensitivity. However, it is particular interesting to look at the two cases of ${ }^{100} \mathrm{Mo}$ and ${ }^{150} \mathrm{Nd}$ in which the experimental $0_{\mathrm{g} . s .}^{+}$and $0_{1}^{+}$half-life values can be directly compared to the models. The IBM-2 half-lives as discussed above do not describe the experimental observation. In fact the prediction is a factor of $37\left({ }^{100} \mathrm{Mo}\right)$ and $14\left({ }^{150} \mathrm{Nd}\right)$ larger than the observed half-life.

A recent idea to explain this deficiency are intermediate scissor states. Those could potentially increase the rate of $0_{1}^{+}$over $0_{\text {g.s. }}^{+}$transition as shown for ${ }^{154} \mathrm{Gd}$ [25]. Further conclusions are expected soon also for ${ }^{150} \mathrm{Nd}$.

\section{Conclusion}

After the observation of many $2 v \beta \beta$ ground state transitions with half-life between $10^{18-21} \mathrm{yr}, 2 v \beta \beta 0_{1}^{+}$transitions have been observed in ${ }^{100} \mathrm{Mo}$ and ${ }^{150} \mathrm{Nd}$ with $T_{1 / 2}=(7.5 \pm 1.2) \cdot 10^{20} \mathrm{yr}$ and $T_{1 / 2}=\left(1.33_{-0.36}^{+0.63}\right) \cdot 10^{20} \mathrm{yr}$ respectively. The discovery of $0_{1}^{+}$transitions in more isotopes is around the corner with the current generation of $0 v \beta \beta$ experiments. In the near future excited state transitions will help to validate and tune NME calculations for $0 v \beta \beta$.

\section{References}

[1] J. Kotila, F. Iachello, Phys. Rev. C 85, 034316 (2012)

[2] NuDat 2, www.nndc.bnl.gov/nudat2/

[3] M. Redshaw, G. Bollen, M. Brodeur, S. Bustabad, D.L. Lincoln, S.J. Novario, R. Ringle, S. Schwarz, Phys. Rev. C 86, 041306 (2012)

[4] G. Douysset, T. Fritioff, C. Carlberg, I. Bergström, M. Björkhage, Phys. Rev. Lett. 86, 4259 (2001)

[5] D.L. Lincoln, J.D. Holt, G. Bollen, M. Brodeur, S. Bustabad, J. Engel, S.J. Novario, M. Redshaw, R. Ringle, S. Schwarz, Phys. Rev. Lett. 110, 012501 (2013)
[6] G. Audia, A.H. Wapstrab, C. Thibaulta, Nucl. Phys. A 729, 337 (2003)

[7] S. Rahaman, V.V. Elomaa, T. Eronen, J. Hakala, A. Jokinen, J. Julin, A. Kankainen, A. Saastamoinen, J. Suhonen, C. Weber et al., Phys. Lett. B 662, 111 (2008)

[8] D. Fink, J. Barea, D. Beck, K. Blaum, C. Böhm, C. Borgmann, M. Breitenfeldt, F. Herfurth, A. Herlert, J. Kotila et al., Phys. Rev. Lett. 108, 062502 (2012)

[9] S. Rahaman, V.V. Elomaa, T. Eronen, J. Hakala, A. Jokinen, A. Kankainen, J. Rissanen, J. Suhonen, C. Weber, J. Äystö, Phys. Lett. B 703, 412 (2011)

[10] D.A. Nesterenko, K. Blaum, M. Block, C. Droese, S. Eliseev, F. Herfurth, E. Minaya Ramirez, Y.N. Novikov, L. Schweikhard, V.M. Shabaev et al., Phys. Rev. C 86, 044313 (2012)

[11] M. Redshaw, B. Mount, E. Myers, F. Avignone, Phys. Rev. Lett. 102, 212502 (2009)

[12] P.M. McCowan, R.C. Barber, Phys. Rev. C 82, 024603 (2010)

[13] M. Redshaw, E. Wingfield, J. McDaniel, E. Myers, Phys. Rev. Lett. 98, 053003 (2007)

[14] V.S. Kolhinen, T. Eronen, D. Gorelov, J. Hakala, A. Jokinen, A. Kankainen, I.D. Moore, J. Rissanen, A. Saastamoinen, J. Suhonen et al., Phys. Rev. C 82, 022501 (2010)

[15] V.I. Tretyak, Y.G. Zdesenko, Atom. Data Nucl. Data Tabl. 80, 83 (2002)

[16] A.S. Barabash, arXiv (2007), 0710.2194

[17] A.S. Barabash, Phys. Rev. C 81, 035501 (2010)

[18] T.N.. Collaboration, R. Arnold, C. Augier, A.S. Barabash, A. Basharina-Freshville, S. Blondel, S. Blot, M. Bongrand, V. Brudanin, J. Busto et al., Nucl. Phys. A 925, 25 (2014)

[19] A. Barabash, P. Hubert, A. Nachab, V. Umatov, Phys. Rev. C 79, 045501 (2009)

[20] B. Lehnert, K. Zuber, Phys. Lett. B 705, 47 (2011) 
[21] B. Lehnert, K. Zuber, E. Andreotti, M. Hult, Phys. Rev. C 87, 034312 (2013)

[22] E. Andreotti, C. Arnaboldi, F.T. Avignone, M. Balata, I. Bandac, M. Barucci, J.W. Beeman, F. Bellini, C. Brofferio, A. Bryant et al., Phys. Rev. C 85, 045503 (2012)

[23] Y.R. Yen, Ph.D. thesis (2013)

[24] J. Barea, J. Kotila, F. Iachello, Phys. Rev. C 87, 014315 (2013)

[25] J. Beller, N. Pietralla, J. Barea, M. Elvers, J. Endres, C. Fransen, J. Kotila, O. Möller, A. Richter, T.R. Rodríguez et al., Phys. Rev. Lett. 111, 172501 (2013)

[26] M. Aunola, J. Suhonen, Nucl. Phys. A 602, 133 (1996)

[27] J. Toivanen, J. Suhonen, Phys. Rev. C 55, 2314 (1997)

[28] S. Stoica, I. Mihut, Nucl. Phys. A A602, 197 (1996)

[29] A. Bakalyarov, A. Balysh, A.S. Barabash, P. Beneš, C. Briançon, V. Brudanin, P. Čermák, V. Egorov, F. Hubert, P. Hubert et al., JETP Lett. 76, 545 (2002)

[30] The GERDA Collaboration, M. Agostini, M. Allardt, E. Andreotti, A.M. Bakalyarov, M. Balata, I. Bara- banov, M.B. Heider, N. Barros, L. Baudis et al., J. Phys. G: Nucl. Part. Phys. 40, 035110 (2013)

[31] S.I. Vasilev, A.A. Klimenko, S.B. Osetrov, A.A. Smolnikov, JETP Lett. 72, 279 (2000)

[32] J. Suhonen, J. Toivanen, A.S. Barabash, I.A. Vanushin, V.I. Umatov, R. Gurriarán, F. Hubert, P. Hubert, Z Phys A - Particles and Fields 358, 297 (1997)

[33] A.S. Barabash, R. Gurriarán, F. Hubert, P. Hubert, J.L. Reyss, J. Suhonen, V.I. Umatov, J. Phys. G: Nucl. Part. Phys. 22, 487 (1996)

[34] J. Hirsch, O. Castanos, P. Hess, O. Civitarese, Phys. Rev. C 51, 2252 (1995)

[35] R. Winter, Phys.Rev. 85, 687 (1952)

[36] J. Suhonen, Nucl. Phys. A 864, 63 (2011)

[37] A. Piepke, M. Beck, J. Bockholt, D. Glatting, G. Heusser, H.V. Klapdor-Kleingrothaus, B. Maier, F. Petry, U. Schmidt-Rohr, H. Strecker et al., Nucl. Phys. A 577, 493 (1994)

[38] A.S. Barabash, F. Hubert, P. Hubert, V.I. Umatov, Eur. Phys. J. A 11, 143 (2001) 